\title{
Pooling the Evidence at the Patient Level: End of the Bivalirudin Saga?
}

\author{
Davide Capodanno ${ }^{1}$ Dominick J. Angiolillo ${ }^{2}$ \\ ${ }^{1}$ Division of Cardiology, A.O.U. "Policlinico-Vittorio Emanuele," \\ University of Catania, Catania, Italy \\ 2 Division of Cardiology, University of Florida College of Medicine, \\ Jacksonville, Florida, United States
}

Thromb Haemost 2020;120:191-193.

Parenteral anticoagulation is essential during percutaneous coronary intervention (PCI) to decrease the risk of periprocedural thrombotic complications. However, the risk of bleeding complications may overshadow its established benefit, ${ }^{1}$ and risk stratification can be challenging., ${ }^{2,3}$ This caveat may be true especially in the context of "enrichment factors" for bleeding such as the use of femoral vascular access and potent antiplatelet therapies. ${ }^{4}$ In the joint guidelines for myocardial revascularization from the European Society of Cardiology and the European Association for Cardio-Thoracic Surgery, published in 2018, unfractionated heparin is recognized as the reference standard for periprocedural anticoagulation (class I), and a few alternatives with a lower class of recommendation are suggested, including enoxaparin and bivalirudin. ${ }^{5}$

Bivalirudin displays several potential pharmacodynamic and pharmacokinetic advantages over heparins. ${ }^{6}$ Nevertheless, in recent years, its use has been largely resized in magnitude. Several reasons may contribute to this tendency, particularly in countries where the use of bivalirudin was more prevalent. ${ }^{7}$ First, the perception of a bleeding advantage with bivalirudin has become relatively less important in the context of increasing use of radial vascular access and avoidance of upstream infusion of glycoprotein IIb/IIIa inhibitors (GPI). ${ }^{8,9}$ Second, recent trials and meta-analyses conducted in patients with acute coronary syndromes (ACS) have questioned the safety of bivalirudin in light of the observed increase in acute stent thrombosis compared with heparin. ${ }^{10,11}$ Indeed, putting the results of bivalirudin trials into perspective is puzzling due to their inherent differences in design, inclusion criteria, methods, and controls. ${ }^{7}$ With respect to the latter, in particular, major confounding derives from the disparate proportions of GPI used in the heparin arms across the years.

Prior investigations comparing bivalirudin with a combination of heparin and GPI did not allow a clear understanding of the relative merits of bivalirudin compared with heparin, which in turn are best captured by more contemporary inves-

\section{received}

October 17, 2019

accepted after revision

October 17, 2019

\author{
Address for correspondence Dominick J. Angiolillo, MD, PhD, \\ University of Florida College of Medicine-Jacksonville, 655 West 8th \\ Street, Jacksonville, Florida 32209, United States \\ (e-mail: dominick.angiolillo@jax.ufl.edu).
}

tigations. $^{12}$ Study-level meta-analyses have attempted over the years to make the best out of bivalirudin data. ${ }^{11,13,14}$ However, the best that such meta-analyses can actually do to explore sources of inconsistency across trials is to analyze subgroups, run sensitivity analyses, or undertake meta-regressions. Therefore, to get more insights into the true merits of bivalirudin and heparin, pooling of patient-level data is required, which enables more sophisticated efforts including identification of independent predictors and examining the temporal relationship between treatments and outcomes.

In this issue of Thrombosis and Haemostasis, Bikdeli and colleagues ${ }^{15}$ publish the protocol of an ongoing attempt to bring the bivalirudin controversy at rest and dissipate current gaps in knowledge by means of a definitive patient-level meta-analysis. The authors scrutinized the available literature in search of randomized clinical trials comparing bivalirudin-based and heparin-based regimens in patients with acute myocardial infarction, and contacted the original investigators as contributors. Patients were pooled if PCI was attempted (i.e., using a "modified intention-to-treat" approach), which however introduces some distortion in the randomization scheme and the need for correcting potential new confounders by covariate adjustment. Patients were considered only in the trial arms where GPI were used as a bailout measure in the bivalirudin group and at any time in the heparin group. In addition, the investigators restricted the search to studies enrolling at least 1,000 subjects, in an attempt to decrease the number of smaller investigations using disparate definitions of clinical endpoints. Indeed, only outcomes measures of ischemia and thrombotic complications with acceptably similar definitions were considered, and mortality was elected as the primary efficacy endpoint due to its univocal definition. On the other hand, bleeding definitions were potentially less consistent, which led the authors to electively use prognostically equivalent information from either or both Thrombolysis in Myocardial

(c) 2020 Georg Thieme Verlag KG Stuttgart · New York
DOI https://doi.org/ 10.1055/s-0039-3400743. ISSN 0340-6245. 
Infarction major or minor bleeding and Bleeding Academic Research Consortium type 3 or 5 bleeding. As a further potential challenge to the validity of this effort, it should be noted that some trials had different event-adjudication modalities (e.g., clinical event committee or site-reported).

Although the results of the meta-analysis will be disclosed in due course, the authors already share some important preliminary information. A total of 8 trials will be pooled, encompassing more than 27,000 patients out of approximately 39,000 after excluding patients assigned to bivalirudin plus planned GPI, those without enzyme elevation, and those where $\mathrm{PCI}$ was not attempted. The 8 trials cover approximately a decade that goes from 2006 to 2017, where many changes obviously occurred in PCI practice for patients with acute myocardial infarction. The pooled analysis will include two trial of patients with non-ST-segment elevation ACS (ACUITY [Acute Catheterization and Urgent Intervention Triage strategY] and ISAR-REACT 4 [Intracoronary Stenting and Antithrombotic Regimen 4]), ${ }^{16,17}$ three trials of patients with ST-segment elevation ACS (HORIZONS-AMI [Harmonizing Outcomes with Revascularization and Stents in Acute Myocardial Infarction], EUROMAX [European Ambulance Acute Coronary Syndrome Angiography], and HEAT-PPCI [How Effective are Antithrombotic Therapies in Primary PCI]), ${ }^{10,18,19}$ and three trials of patients across the spectrum of ACS (BRIGHT [Bivalirudin foR acute myocardial Infarction underGoing angioplasty in $\mathrm{CHi}$ nese patienTs], MATRIX [Minimizing Adverse Hemorrhagic Events by Transradial Access Site and Systemic Implementation of Angiox], and VALIDATE-SWEDEHEART [Bivalirudin versus Heparin in ST-Segment and Non-ST-Segment Elevation Myocardial Infarction in Patients on Modern Antiplatelet Therapy on the Swedish Web-system for Enhancement and Development of Evidence-based care in Heart disease Evaluated According to Recommended Therapies]) (- Fig. 1) ${ }^{20-22}$ Pooling these trials at the patient level will give important insights not only on the relative merits of bivalirudin and heparin but also

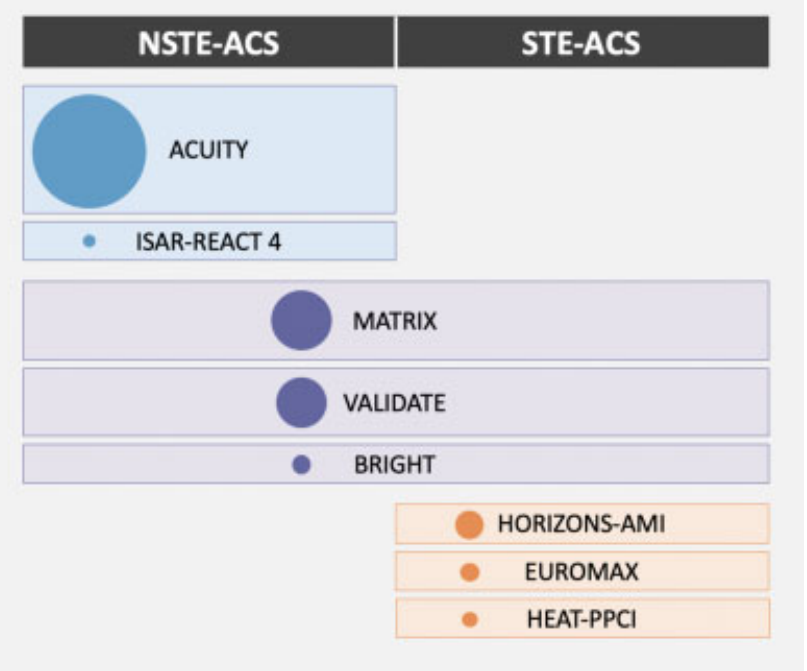

Fig. 1 Trials of bivalirudin across the spectrum of acute coronary syndromes included in the individual patient-level meta-analysis from Bikdeli and colleagues. ${ }^{15}$ Size of the circles is proportional to the number of patients included in the trials. on the treatment interaction with important variables such as clinical presentation with non-ST-segment-elevation ACS or ST-segment-elevation ACS, vascular access, and sex.

It goes without saying that when such sophisticated approaches are attempted, the amount of preparatory work before running any analysis is remarkable. This includes obtaining the individual data sets, collecting data centrally, identifying potential errors, several iterative revisions, and querying the original investigators multiple times to collect missing data, finalize the acquisition, and lock the pooled data set. Notably, this effort took 4 years to be completed, but the bivalirudin saga hopefully seems close to an end. As such, the authors should be commended for embarking into this kind of complex endeavor, which conceptually will place their meta-analysis at the highest place in the hierarchy of evidence.

\section{Conflict of Interest}

D. C. reports speaker's and consulting fees from AstraZeneca, Bayer, Boehringer Ingelheim, Daiichi Sankyo, Sanofi. D.J.A. reports grants and personal fees from Amgen, Aralez, Bayer, Biosensors, Boehringer Ingelheim, Bristol-Myers Squibb, Chiesi, Daiichi-Sankyo, Eli Lilly, Janssen, Merck, Sanofi, CeloNova, and Astra Zeneca; personal fees from Haemonetics, PhaseBio, PLx Pharma, Pfizer, The Medicines company, and St Jude Medical; grants from CSL Behring, Eisai, Gilead, Idorsia Pharmaceuticals Ltd, Matsutani Chemical Industry Co., Novartis, Osprey Medical, Renal Guard Solutions, Scott R. MacKenzie Foundation, NIH/NCATS Clinical and Translational Science Award to the University of Florida UL1 TR000064 and NIH/NHGRI U01 HG007269, outside the submitted work.

\section{References}

1 Angiolillo DJ, Rollini F, Franchi F. The quest for the optimal periprocedural antithrombotic treatment strategy in ACS patients undergoing PCI. J Am Coll Cardiol 2018;71(11):1243-1245

2 Capodanno D, Greco A. Risk stratification for bleeding in the elderly with acute coronary syndrome: not so simple. Thromb Haemost 2018;118(06):949-952

3 Biancari F, Brascia D, Onorati F, et al. Prediction of severe bleeding after coronary surgery: the WILL-BLEED Risk Score. Thromb Haemost 2017;117(03):445-456

4 Singh M. Bleeding avoidance strategies during percutaneous coronary interventions. J Am Coll Cardiol 2015;65(20):2225-2238

5 Neumann F-J, Sousa-Uva M, Ahlsson A, et al; ESC Scientific Document Group. 2018 ESC/EACTS Guidelines on myocardial revascularization. Eur Heart J 2019;40(02):87-165

6 Weitz JI, Harenberg J. New developments in anticoagulants: past, present and future. Thromb Haemost 2017;117(07):1283-1288

7 Capodanno D, De Caterina R. Bivalirudin for acute coronary syndromes: premises, promises and doubts. Thromb Haemost 2015;113(04):698-707

8 Perdoncin E, Seth M, Dixon S, et al. The comparative efficacy of bivalirudin is markedly attenuated by use of radial access: insights from Blue Cross Blue Shield of Michigan Cardiovascular Consortium. Eur Heart J 2016;37(24):1902-1909

9 Angiolillo DJ. Vascular access and antithrombotic therapy in patients with acute coronary syndrome. Lancet 2018;392(10150):801-802

10 Shahzad A, Kemp I, Mars C, et al; HEAT-PPCI trial investigators. Unfractionated heparin versus bivalirudin in primary percutaneous 
coronary intervention (HEAT-PPCI): an open-label, single centre, randomised controlled trial. Lancet 2014;384(9957):1849-1858

11 Capodanno D, Gargiulo G, Capranzano P, Mehran R, Tamburino C, Stone GW. Bivalirudin versus heparin with or without glycoprotein IIb/IIIa inhibitors in patients with STEMI undergoing primary PCI: an updated meta-analysis of 10,350 patients from five randomized clinical trials. Eur Heart J Acute Cardiovasc Care 2016;5(03):253-262

12 Elgendy IY, Capodanno D. Heparin versus bivalirudin for percutaneous coronary intervention: has the debate come to an end? J Thorac Dis 2017;9(11):4305-4307

13 Cavender MA, Sabatine MS. Bivalirudin versus heparin in patients planned for percutaneous coronary intervention: a meta-analysis of randomised controlled trials. Lancet 2014;384(9943):599-606

14 Navarese EP, Schulze V, Andreotti F, et al. Comprehensive metaanalysis of safety and efficacy of bivalirudin versus heparin with or without routine glycoprotein IIb/IIla inhibitors in patients with acute coronary syndrome. JACC Cardiovasc Interv 2015;8(1 Pt B):201-213

15 Bikdeli B, McAndrew T, Crowley A, et al. Individual patient data pooled analysis of randomized trials of bivalirudin versus heparin in acute myocardial infarction: rationale and methodology. Thromb Haemost 2019;120(02):348-361
16 Stone GW, McLaurin BT, Cox DA, et al; ACUITY Investigators. Bivalirudin for patients with acute coronary syndromes. N Engl J Med 2006;355(21):2203-2216

17 Kastrati A, Neumann F-J, Schulz S, et al; ISAR-REACT 4 Trial Investigators. Abciximab and heparin versus bivalirudin for non-ST-elevation myocardial infarction. N Engl J Med 2011;365 (21):1980-1989

18 Stone GW, Witzenbichler B, Guagliumi G, et al; HORIZONS-AMI Trial Investigators. Bivalirudin during primary $\mathrm{PCI}$ in acute myocardial infarction. N Engl J Med 2008;358(21):2218-2230

19 Steg PG, van 't Hof A, Hamm CW, et al; EUROMAX Investigators. Bivalirudin started during emergency transport for primary $\mathrm{PCI}$. N Engl J Med 2013;369(23):2207-2217

20 Han Y, Guo J, Zheng Y, et al; BRIGHT Investigators. Bivalirudin vs heparin with or without tirofiban during primary percutaneous coronary intervention in acute myocardial infarction: the BRIGHT randomized clinical trial. JAMA 2015;313(13):1336-1346

21 Valgimigli M, Frigoli E, Leonardi S, et al; MATRIX Investigators. Bivalirudin or unfractionated heparin in acute coronary syndromes. N Engl J Med 2015;373(11):997-1009

22 Erlinge D, Omerovic E, Fröbert O, et al. Bivalirudin versus heparin monotherapy in myocardial infarction. N Engl J Med 2017;377 (12):1132-1142 